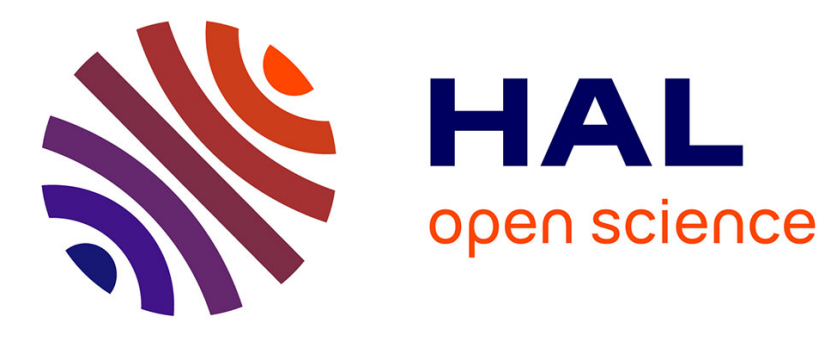

\title{
Methodology of tolerance synthesis using bond graph
} van Hoa Nguyen, Damien Eberard, Wilfrid Marquis-Favre, Laurent

\author{
Krähenbühl
}

\section{To cite this version:}

van Hoa Nguyen, Damien Eberard, Wilfrid Marquis-Favre, Laurent Krähenbühl. Methodology of tolerance synthesis using bond graph. ECMS European Conference on Modelling and Simulation, May 2013, Ålesund, Norway. pp.\#143. hal-00799531

\section{HAL Id: hal-00799531 \\ https://hal.science/hal-00799531}

Submitted on 15 Apr 2019

HAL is a multi-disciplinary open access archive for the deposit and dissemination of scientific research documents, whether they are published or not. The documents may come from teaching and research institutions in France or abroad, or from public or private research centers.
L'archive ouverte pluridisciplinaire HAL, est destinée au dépôt et à la diffusion de documents scientifiques de niveau recherche, publiés ou non, émanant des établissements d'enseignement et de recherche français ou étrangers, des laboratoires publics ou privés. 


\title{
Methodology of tolerance synthesis using bond graph
}

\author{
Van Hoa NGUYEN ${ }^{+}$, Damien EBERARD ${ }^{+}$, Wilfrid MARQUIS-FAVRE ${ }^{+}$, Laurent KRAHENBUHL* \\ + Université de Lyon, INSA de Lyon, Laboratoire Ampère (CNRS UMR5005), \\ Address: 20 Avenue Albert Einstein 69621 Villeurbanne cedex - France, \\ Email: surname.name@insa-lyon.fr \\ *Université de Lyon, École Centrale de Lyon, Laboratoire Ampère (CNRS UMR5005) \\ Address: 36 avenue Guy de Collongue - 69134 Ecully Cedex - France, \\ Email: laurent.krahenbuhl@ec-lyon.fr
}

\begin{abstract}
KEYWORDS
Tolerance synthesis, Bond graph, Inverse model, Aleatory uncertainty, Probability theory

ABSTRACT

This paper presents a methodology of parametric tolerance synthesis with respect to output aleatory uncertainty specifications. It relies on density function propagation through the inverse model. The resulting parameter density function is then used to synthesize a confidence interval suitable for sizing purpose. As an illustration, parametric tolerance synthesis on a DC motor rotating a load is processed.
\end{abstract}

\section{INTRODUCTION}

Nowadays, mechatronics touches a wide range of applications, in daily life as well as in industry. Following technologies improvements, design process has to balance time development and manufacturing cost while systems requirements become increasingly sharp. Part of this process is concerned with parametric tolerance intended for manufacturers towards minimal cost components design with respect to requirements.

In the framework of this paper, we are interested in the problem of parametric tolerance synthesis with respect to specifications including output aleatory uncertainties. The aim is to obtain the tolerance of the design parameter to keep satisfying the requirement. We adopt the bond graph language (for system modelling, structural analysis and inverse model generation) and probability tools (for aleatory uncertainties representation and propagation).

Classically represented by its transfer function, or state equations, mechatronic system are often treated as a whole, without consideration to local physical phenomena. We propose, in this paper, to use the bond graph language [1] to represent the mechatronic system, in order to better adapt the modelling to physical phenomena and causalities. Bond graph is a multi-discipline language, which facilitates the representation of multi-domain mechatronic system. It is popularly used in engineering applications [2,3], especially multi-domain physical systems [4,5]. A mathematical foundation of bond graph is established in [6].

The bond graph framework provides users with model inversion algorithms [7, 8]. Based on the concept of bi- causality [9], the bond graph model serves for design purposes while keeping the same structure as that of the direct model. Inverse model approach reduces the calculus time and the number of simulating iterations, while, at the same time, takes a good view on physical phenomena and effects. The structural invertibility of a bond graph model is easily checked thanks to existing procedures based on power lines [8] and causal paths [10].

Uncertainty in a mechatronic system is categorized into three distinct classes: variability, uncertainty, and error [11]. Different approaches for uncertainty characterizing and managing are required for the different classes. In this paper we focus on aleatory uncertainty. A similar procedure to deal with epistemic uncertainty in mechatronic system tolerance synthesis has been treated in [12].

Aleatory uncertainty in a mechatronic system refers to the inherent variation associated with the physical system or the environment. In the simplest form, aleatory uncertainty is often quantified with interval arithmetic [13, 14]. However, it is proven to be expensive in computation time and the propagation result is often over-estimated. Therefore, aleatory uncertainty is usually represented by probability theory in the form of probability density function (PDF).

Having the inverse model and uncertainties on output specifications, one has to propagate them to the design parameters. In order to propagate aleatory uncertainty through a mechatronic model, there are several popular approaches: the classical Monte-Carlo and Polynomial Chaos. MonteCarlo is a computational sampling and simulation methods. A general overview of this method is given in [15]. Polynomial Chaos is based on the solution of stochastic differential equations. Firstly developed in [16], this method was continuously refined and applied into structural analysis problems [17-20]. The cost of polynomial chaos method is much cheaper than Monte-Carlo, yet is still significantly high.

Based on the principle of conservation of probability, a stochastic formulation of physical system was proposed in [21]. That formulation was applied to the inverse model to propagate the PDFs [22] and to form the probabilistic bond graph. This method takes into account the dynamics of stochastic systems and can be applied to an inverse model. However, it requires to determine firstly the joint PDF of the energy variables and their derivatives, which is 
sometimes hard to obtain.

In this paper, we shall use the probability density function to represent output uncertainties specifications. Moreover, uncertainty propagation through inverse model is processed using an analytical way.

The paper is organized as follows. In the next section, the problem of tolerance synthesis is briefly formulated. In the third section, a methodology of tolerance synthesis is presented. The methodology is illustrated in the fourth part by an example of tolerance synthesis on a DC motor where both cases of output mono-uncertainty and output multiuncertainty are considered.

\section{Formulation OF THE PROBLEM}

Our goal is to determine the tolerances of design parameters given the probabilistic representation of aleatory output uncertainties.

System's specifications contain the deterministic behaviour that characterizes the (ideal) scenario to be followed. The associated set of desired output trajectories is then considered to be subject to aleatory uncertainties. Output uncertainties are translated into a family of trajectories living in the neighbourhood of the desired ones. The model gives us the relation between the output behaviours and the design parameters, which sets a base of knowledge for tolerance synthesis.

Let us consider a given mechatronic system. We shall use probability density functions to quantify the output aleatory uncertainties included in the specifications. It describes the relative likelihood for the output to behave in a given way. Once the aleatory output behaviour is quantified with PDFs, the aim is to link it with the design parameters. The inverse model gives us this link with an explicit relation between outputs and design parameters. The PDF of the output uncertainty is then propagated through the inverse model using theorem 1 [22]:

Theorem 1: Let $\Theta_{1}$ be an aleatory variable with marginal PDF $\phi_{\Theta_{1}}\left(\theta_{1}\right)$, and $\Theta_{2}$ another aleatory variable such that $\Theta_{2}=g\left(\Theta_{1}\right)$ where $g$ is a diffeomorphism. Then the associated PDF $\phi_{\Theta_{2}}\left(\theta_{2}\right)$ is given by

$$
\phi_{\Theta_{2}}\left(\theta_{2}\right)=\frac{\phi_{\Theta_{1}}\left(g^{-1}\left(\theta_{2}\right)\right)}{\left|g^{\prime}\left(g^{-1}\left(\theta_{2}\right)\right)\right|},
$$

where $g^{-1}$ is the inverse function of $g$ and $g^{\prime}$ its differential.

The latter remains true for a vector of aleatory variables.

Moreover, notice that when $g$ is not a global differomorphism (a popular scenario) but satisfies some smoothness condition, the $\theta_{1}$-space can be partitioned in such a way that $g$ restricted to any subpart is a local diffeomophism denoted $g_{i}$. In that case, the marginal PDF of $\Theta_{2}$ is given by

$$
\phi_{\Theta_{2}}\left(\theta_{2}\right)=\sum_{i=1}^{k} \frac{\phi_{\Theta_{1}}\left(g_{i}^{-1}\left(\theta_{2}\right)\right)}{\left|g^{\prime}\left(g_{i}^{-1}\left(\theta_{2}\right)\right)\right|} .
$$

In practice, one not only consider a single variable but rather a vector of aleatory variables. In that case, the aleatory uncertainties are represented by the joint PDFs of those vectors and the propagation is actualized on those joint PDFs.
The above theorem assumes that the vectors of aleatory variables $\Theta_{1}$ and $\Theta_{2}$ have the same dimension. If it is not the case, fictive variables have to be added in order to complete the dimension.

Propagating the uncertainty in output behaviours yields the probability density functions of the design parameters, which allow the determination of tolerances of design parameters with respect to the specifications.

\section{METHODOLOGY OF TOLERANCE SYNTHESIS IN THE PRESENCE OF ALEATORY UNCERTAINTY}

We propose the next procedure to solve our problem of tolerance synthesis:

A Modelling: Construct the bond graph of the system, model the aleatory uncertainties included in the output specifications with probability density function, determine the set of design parameters and outputs.

B Adequacy: Check the adequacy between the system structure and the input/output specifications [22].

C Inversion: Test the structural invertibility [8, 10] and construct the inverse model [22,23]

D Propagation: Deduce the PDF associated with the design parameters by propagating the output uncertainties through the inverse model.

E Tolerance synthesis: Synthesize the tolerance of the design parameters from their computed uncertainties.

F Validation: Verify the chosen tolerance in simulation in direct model.

\section{A. Modelling}

The bond graph model is constructed based on the physical phenomena of the deterministic mechatronic system. Because we focus on the problem of tolerance synthesis, the structure of the model is assumed to be known and fixed (which means that there is no black-box, models commutation, discontinuity). The parameters of system are then classified into two sets: the set of known parameters and the set of design parameters (those of interest for tolerance synthesis).

Output uncertainties are modelled with their probability density functions.

\section{B. Adequacy verification}

Depending on its nature, some types of specifications are not compatible with certain types of dynamic systems or model workspace, etc.... A verification of adequacy between the system's structure and the input/output specifications is therefore essential. In the case of linear system, an adequacy verification procedure based on the order of system is given in [22]. This step means to avoid unnecessary further advancement, if the structure of system is not adequate with the demanded specifications. It gives also hints to redefine a proper set of requirements in that case.

\section{Inversion}

We shall deduce an explicit relation between the outputs and the design parameters via the inverse bond graph model. The direct model outputs will be the inputs of the inverse model and the design parameters will be the outputs of the inverse model. A structural analysis of causal paths and 
power lines $[22,23]$ has to be done to verify the invertibility. The inverse model of minimum order can be found from the bicausal bond graph with the procedure detailed in [22]. It is necessary to find the model of minimum order, because it decides the order of derivatives of outputs in the inverse model, hence, in the calculation cost.

\section{Propagation}

There are two ways to propagate the uncertainties throughout the inverse bond graph model: global approach and local approach. In global approach, the system is considered as a whole and the output (or vector of outputs) is directly connected to the parameter(s) by a global relation (or a set of relations); the uncertainties on output (or vector of outputs) is propagated to the parameter(s) under interest by applying the theorem 1 to that global relation (or a set of relations). Another approach is the local propagation: PDFs from outputs (or vector of outputs) are transferred from element to element in the (inverse) bond graph model, until it reaches the design parameters. This approach offers a closer look to the effects of uncertainty to local physical phenomena.

In case of output multi-uncertainty, the obtained PDF is the joint PDF of design parameters (including "fictive" parameters, if they were added to balance the vector dimension). In order to determine the marginal PDF of one design parameter, we need to integrate the joint PDF on all the domains of definition of the other parameters (including the "fictive" variables) following theorem 2 below.

Theorem 2: Given the joint PDF $\phi_{\left(\Theta_{1}, \Theta_{2}, \ldots, \Theta_{n}\right)}$, the marginal PDF of an element $\theta_{1}$ is calculated:

$\phi_{\Theta_{1}}\left(\theta_{1}\right)=\int_{\Theta_{2}} \ldots \int_{\Theta_{n}} \phi_{\left(\Theta_{1}, \Theta_{2}, \ldots, \Theta_{n}\right)}\left(\theta_{1}, \theta_{2}, \ldots, \theta_{n}\right) d \theta_{2} \ldots d \theta_{n}$

\section{E. Tolerance synthesis}

The final PDF obtained for the design parameter provides us with information on its distribution law. Different from interval analysis, where we obtain only the "worst case solution", with the PDF, we obtain more information about the distributing interval of the design parameters. This PDF is, however, not necessarily Gaussian nor symmetric, which is required in most manufacturing processes. An adaptation step is therefore necessary. In the frame of our research, the tolerances of the design parameters are defined as a Gaussian laws with the same mean and variance as the computed PDF.

\section{F. Validation}

From the obtained tolerance, we generate a number of samples, following the synthesized PDF of the parameter, and re-inject them into the direct bond graph model. Simulation is made with the generated values to verify the specifications on system's performance. The simulated results are checked with the set of requirements. Since the initial specifications are given in the form of PDFs, on may have to compare the PDF obtained from simulation's results and initial PDFs.

This can be done visually if the PDF obtained from simulations stays totally inside the interval of the initial PDF.
However, in some case, the comparison is hard to be done visually, a numeric criterion is therefore needed. We adopt here the criterion of quantiles:

Definition 1: The quantile function $Q_{X}$ at level $u$ is defined by $Q_{X}(u)=\inf \left\{x / F_{X}(x) \geq u\right\}$ where $F_{X}$ is the distribution function of the aleatory variable $X$.

The two PDFs are considered similar enough if the error between them is less than $15 \%$ :

Criterion 1: The criterion of quantiles gives the mean average error (MAE) as follows

$$
M A E=\Sigma \frac{\left|Q_{i}^{1}-Q_{i}^{2}\right|}{\sigma^{1}} \leq 15 \%
$$

with $i=\{1,5,25,50,75,95,99\} \%, \sigma^{1}$ is the variance of the first PDF.

In the next part, we illustrate our proposed procedure on the example of a DC motor rotating a load.

\section{EXAMPLE}

Consider a DC motor rotating a load (Figure 1). As an illustration of our methodology, we process the tolerance synthesis of the internal resistance of the DC motor. We shall study a simple output mono-uncertainty case and an output multi-uncertainty case.

Modelling assumptions: The electrical part contains a voltage source $u$, an internal resistance $R$ and an inductance $L$. The (ideal) electromechanical coupling is characterized by a torque constant $k_{c}$. The mechanical part takes into account the motor axis inertia $J_{m}$ and the load inertia $J_{c}$, a reduction gear ratio $1 / N$ and the viscous friction coefficient $b_{c}$ on the load axis.

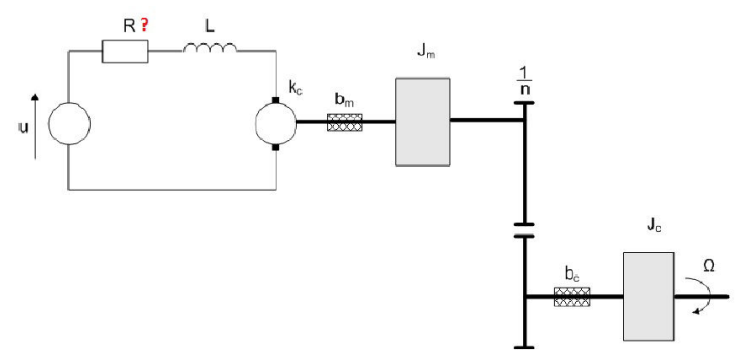

Fig. 1: Scheme of a DC motor rotating a load

\section{Data specifications.}

$L \quad$ Motor internal self inductance

$k_{c} \quad$ Electromechanical coupling

$J_{m} \quad$ Motor axis inertia

$N \quad$ Gear ratio

$J_{c} \quad$ Load inertia

$b_{c} \quad$ Viscous friction coefficient

$u$ Input voltage

$$
\begin{gathered}
0.001[\mathrm{H}] \\
0.031[\mathrm{~N} . \mathrm{m} / \mathrm{A}] \\
1.8 \times 10^{-6}\left[\mathrm{~kg} . \mathrm{m}^{2}\right] \\
1 / 20 \\
2 \times 10^{-4}\left[\mathrm{~kg} . \mathrm{m}^{2}\right] \\
0.0001\left[\mathrm{~N} . \mathrm{m} / \mathrm{rad} . \mathrm{s}^{-1}\right] \\
20[\mathrm{~V}]
\end{gathered}
$$

Performance specifications. The output angular velocity $\Omega$ is desired to follow a second order step response with an amplitude $K=32$, a damping ratio $\xi=24$ and an undamped frequency $\omega_{n}=650$ rad. $s^{-1}$.

Uncertainty specifications. The stationary output velocity may vary in the interval $\delta K= \pm 1$. For the other two parameters, one specifies $\delta \xi= \pm 3$ and $\delta \omega_{n}= \pm 30 \mathrm{~s}^{-1}$. 
These variations form an envelop that the output trajectory is expected to lie within.

We shall first study the mono-uncertainty case where only uncertainty on the amplitude $K$ is taken into account. Then, we study the multi-uncertainty case where variations on $K, \xi$ and $\omega_{n}$ are considered.

\section{Mono-uncertainty}

Modelling. The bond graph model of the system is given in Fig. 2. The internal motor resistance $R$ is the design

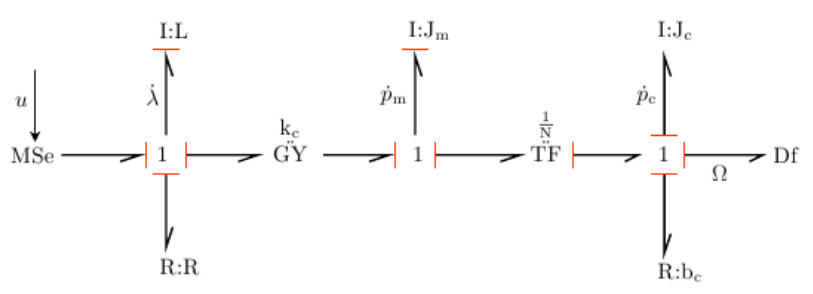

Fig. 2: Causal bond graph representation of a DC motor rotating a load

parameter and the output is the angular velocity $\Omega$.

We model the uncertainty on $K$ as a Gaussian distribution with density probability function $\phi_{K}$, which has as expectation the nominal deterministic value $\mu_{K}=32$ and variance $\sigma_{K}=1 / 3$.

$$
\phi_{K}=\mathcal{N}\left(\mu_{K}, \sigma_{K}\right)
$$

Adequacy. We note $J=\frac{J_{m}}{n}+n J_{c}$ and $b=\frac{b_{m}}{n}+n b_{c}$. The dynamic response writes

$$
\left(\frac{k_{c}}{n}+\frac{R b}{k_{c}}\right) \Omega+\left(\frac{R J+L b}{k_{c}}\right) \dot{\Omega}+\frac{L J}{k_{c}} \ddot{\Omega}=u
$$

The considered model is therefore a second order. As a result, its structure is in adequacy with the specification.

Inverse model. The structure of system verifies the criterion of invertibility [23] from the output $\Omega$ to the design parameter $R$. The bicausal bond graph model is given in Fig. 3.

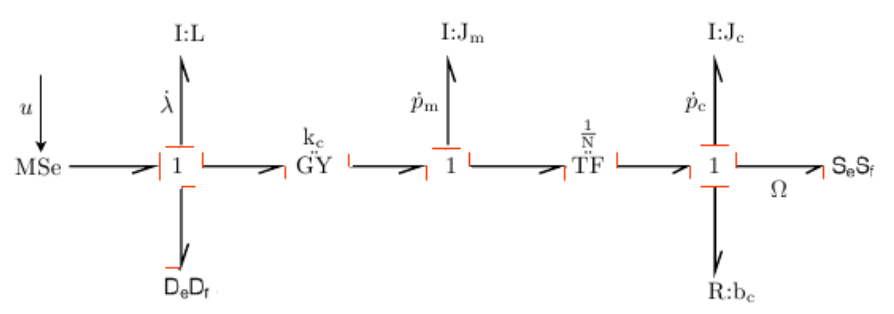

Fig. 3: Bicausal bond graph representation of inverse model

The minimum order inverse model, obtained from [22] applied to the model depicted in Figure 3, is given by

$$
\begin{aligned}
R & =\frac{u-L\left[\frac{1}{k_{c}}\left(\frac{J_{m}}{n}+n J_{c}\right) \ddot{\Omega}-\frac{1}{k_{c}}\left(\frac{b_{m}}{n}+n b_{c}\right) \dot{\Omega}\right]-\frac{k_{c}}{n} \Omega}{\frac{1}{k_{c}}\left(\frac{J_{m}}{n}+n J_{c}\right) \dot{\Omega}+\frac{1}{k_{c}}\left(\frac{b_{m}}{n}+n b_{c}\right) \Omega} \\
& =g(u, \Omega, \dot{\Omega}, \ddot{\Omega}) \\
& =h\left(u, K, \xi, \omega_{n}\right) .
\end{aligned}
$$

Propagation. Uncertainty on the amplitude $K$ is propagated to the internal resistance $R$ through the inverse model $h$ given by (2). This results in the $\operatorname{PDF} \phi_{R}$ which represents the uncertainty on the design parameter $R$ evolving in time (Fig. 4).

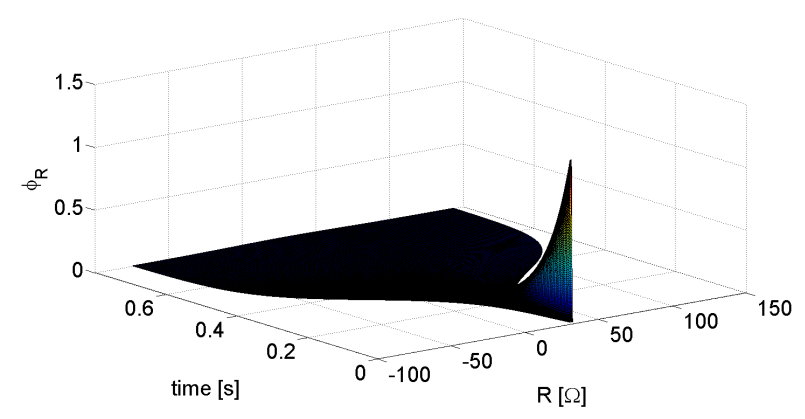

Fig. 4: $\phi_{R}$ evolving in time

Tolerance synthesis. Projecting the obtained PDF set onto $(R, t)$, we obtain the set of values of $r$ with $\phi_{R}(r)>0$ and the curve of modal value of $R$ (Fig. 5). We want the

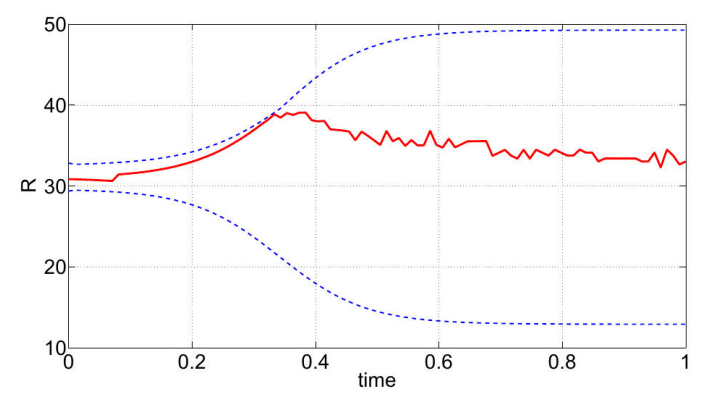

Fig. 5: Set of values of $r$ with $\phi_{R}(r)>0$ and modal value of $R$

output trajectories to be bounded by the specified envelop (blue dotted curve). The value of $R$ is therefore supposed to stay inside the interval defined by the minimum of the upper curve and the maximum of lower curve in Fig. 5. However, since the amplitude uncertainty mainly acts on the steadystate values of the response and little on the transient response, we shall determine the tolerance of $R$ from the PDF at an ad hoc time. In this example, we have arbitrary chosen 0.1 second. The output trajectory is therefore expected to stay inside our envelop from 0.1 second. This PDF is however, not necessarily Gaussian, we choose therefore the PDF of the tolerance as an Gaussian with same mean and variance.

Validation. We generate random samples of $R$ according to the PDF tolerance and re-inject into our direct model. 
The simulations give us the corresponding gains and trajectories (Fig. 6). As predicted, the trajectories go out of the

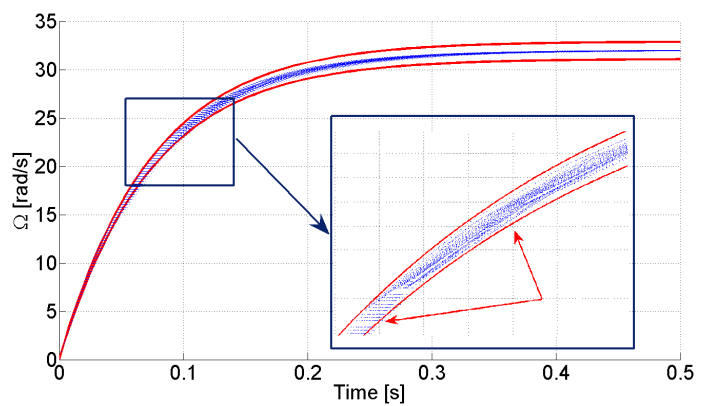

Fig. 6: Simulations with the synthesized tolerance

envelop for a short time, but they do lie inside the envelop from 0.1s (Fig. 6). The PDFs of $K$ are synthesized from the obtained samples. We notice also that the PDF of $K$ ob-

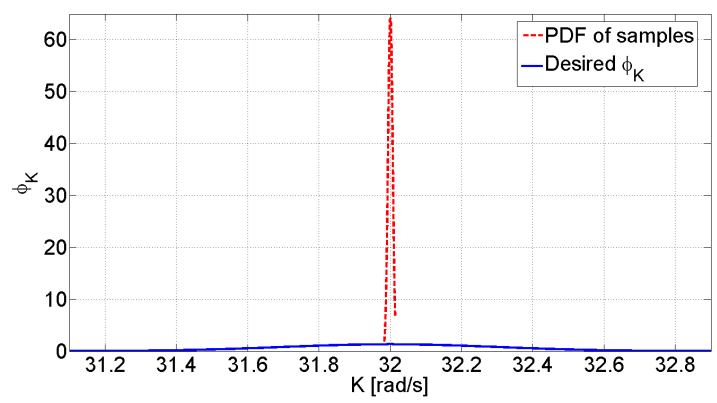

Fig. 7: Desired PDF of $K$ and obtained PDF from samples

tained from simulated samples is much more concentrated to the nominal value than the desired PDF (Fig. 7). The specifications on $K$ is met. We can therefore validate our choice of tolerance on $R$

\section{Output multi-uncertainty}

Modelling. The modelling assumptions are identical to the previous case. We thus deal with the same bond graph model given in Fig. 2. The only difference resides in the fact that we now add the damping ratio $\xi$ and the undamped frequency $\omega_{n}$ uncertainties in order to take into account transient response uncertainties. Similarly, the uncertainties on the undamped natural frequency $\omega_{n}$ and on the damping ratio $\xi$ are represented by their PDFs (assumed to be Gaussian)

$\phi_{K}=\mathcal{N}(32,0.33), \quad \phi_{\xi}=\mathcal{N}(24,1), \quad \phi_{\omega_{n}}=\mathcal{N}(650,10)$.

The steps of adequacy verification and inversion are identical to the previous case.

Propagation. The uncertainty is given on a vector $\left[\begin{array}{l}K \\ \omega_{n} \\ \xi\end{array}\right]$. In order to propagate, we need to add, for instance, $K$ and $\omega_{n}$ (as fictive variables) to $R$ to form a vector. The uncertainty vector is then $\left[\begin{array}{c}R \\ K \\ \omega_{n}\end{array}\right]$ and we have the relation

$$
\left[\begin{array}{l}
R \\
K \\
\omega_{n}
\end{array}\right]=G\left[\begin{array}{l}
K \\
\omega_{n} \\
\xi
\end{array}\right]
$$

where the linear map is given by $G=\left(g, i d_{K}, i d_{\omega_{n}}\right)$. We assume that $K, \omega_{n}$ and $\xi$ are independent variables. From independency, the joint PDF of $\left(K, \omega_{n}, \xi\right)$ is the product of marginal PDFs and is calculated as

$$
\phi_{\left(K, \omega_{n}, \xi\right)}=\phi_{K} \cdot \phi_{\omega_{n}} \cdot \phi_{\xi}
$$

Thefore, the Jacobian of $G$, denoted $\left|J_{G}\right|$ is

$$
\left|J_{G}\right|=\operatorname{det}\left[\begin{array}{ccc}
\frac{\partial g\left(K, \omega_{n}, \xi\right)}{\partial K} & \frac{\partial g\left(K, \omega_{n}, \xi\right)}{\partial \omega_{n}} & \frac{\partial g\left(K, \omega_{n}, \xi\right)}{\partial \xi} \\
\frac{\partial K}{\partial K} & 0 & 0 \\
0 & \frac{\partial \omega_{n}}{\partial \omega_{n}} & 0
\end{array}\right]
$$

The joint PDF of $\left(R, K, \omega_{n}\right)$ is then computed as (following Theorem 1) as

$$
\phi_{\left(R, K, \omega_{n}\right)}=\left|J_{G}\right|^{-1} \cdot \phi_{\left(K, \omega_{n}, \xi\right)}
$$

The PDF of $R$ is deduced from the joint PDF of $(R, K)$ while integrating along $K$ and $\omega_{n}$ (see Theorem 2). The obtained PDF, depicted in Figure 8, represents the variability of $R$ evolving in time.

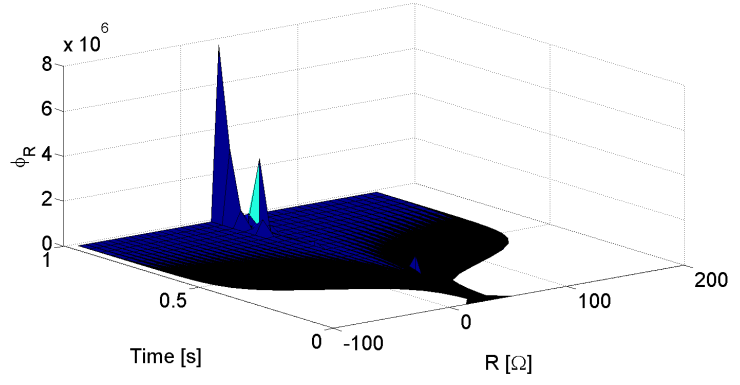

Fig. 8: $\phi_{R}$ evolving in time

Tolerance synthesis. Projecting this PDF set to $(R, t)$, we obtain the set of values of $r$ with $\phi_{R}(r)>0$ and the curve of modal value of $R$ (Fig. 9) The tolerance of $R$ is

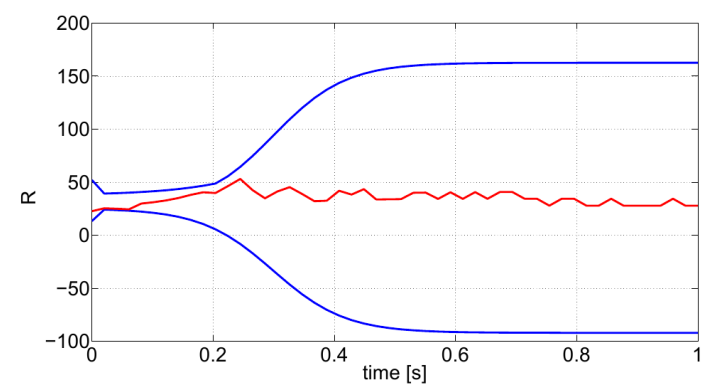

Fig. 9: Set of values of $r$ with $\phi_{R}(r)>0$ and modal value of $R$

therefore synthesized as PDF that gives the smallest interval of $R$ with $\phi_{R}(r)>0$ in $t$.

Validation We generate random samples of $R$ according to the PDF tolerance and re-inject into our direct model. The 


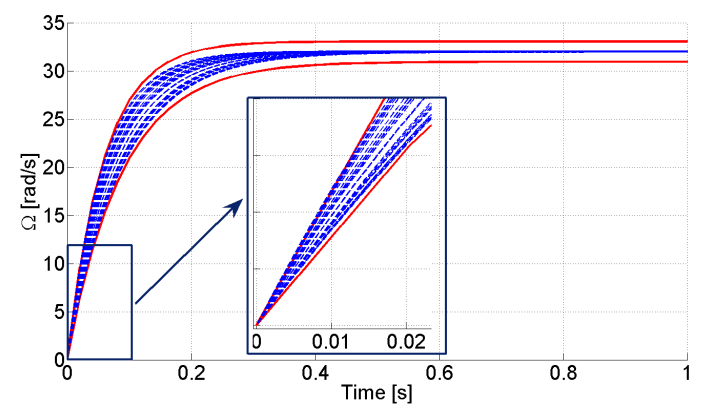

Fig. 10: Simulations with the synthesized tolerance

simulation gives us the corresponding gains and trajectories (Fig. 10). A tiny part of the trajectories goes out of the envelop before $0.01 \mathrm{sec}$. However, it is numerical error due to the fact that we began the simulation at $0.01 \mathrm{sec}$, and can be eliminated by setting the start point of simulation closer to 0 . We verify also the PDFs of $K, \omega_{n}, \xi$ computed from the simulated samples. They are all much more concentrated to the nominal value than the demanded PDF, therefore verified. We present here only the PDF of $K$ (Fig. 11). The

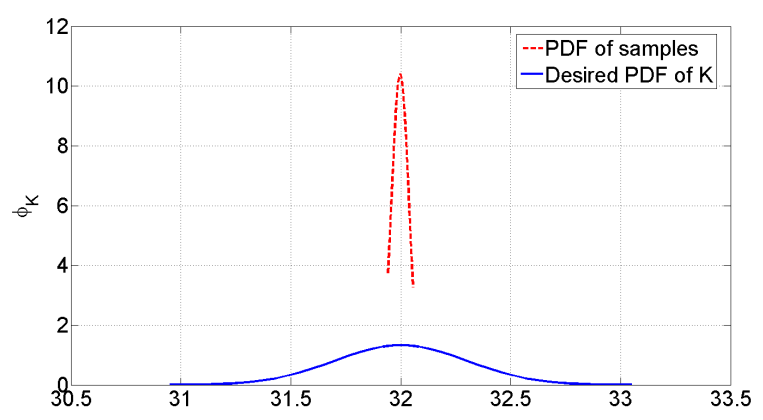

Fig. 11: Simulations with the synthesized tolerance

specifications are met. We can therefore validate our choice of tolerance on $R$

\section{CONCLUSION AND OUTLOOK}

In this paper, a methodology to address the problem of parametric tolerance synthesis in the presence of aleatory uncertainties has been presented.

Bond graph was chosen as the modelling tool, because of its multi-disciplinary and acausality. The inverse approach was adopted. That methodology proves its interest over the direct approach in term of calculation cost. However, it requires the structure of system to be invertible.

The proposed methodology provides users with the probability density function of the design parameter, evolving in time, which offers a complete view on the parameter's distribution. The methodology was illustrated on a simple example of tolerance synthesis on a DC motor.

As we mentioned above, three types of uncertainties coexist in mechatronic systems, which, in general, can not be treated separately. A combined representation and resolution should be investigated. Another perspective concerns stochastic uncertain systems which demand to handle much more complex specifications.

\section{REFERENCES}

[1] H. Paynter, Analysis and Design of Engineering Systems, ISBN.0262160048 ed. Cambridge, USA: The M.I.T Press, 1961.

[2] P. C. Breedveld, "Modeling and simulation of dynamic systems using bond graphs," In Control Systems, Robotics and Automation, from Encyclopedia of Life Support Systems (EOLSS), Developed under the Auspices of the UNESCO. EOLSS Publishers, p. 1-36, 2008.

[3] D. Karnopp, D. Margolis, and R. Rosenberg, System Dynamics. Modeling and Simulation of Mechatronic systems, 4th ed. Hoboken, New Jersey: John Wiley \& sons, Inc, 2012.

[4] P. Breedveld, "Port-based modelling of multidomain physical systems in terms of bond graphs," Simulation Techniques for Applied Dynamics, p. 141-190, 2009.

[5] D. Karnopp, "Bond graph models for electrochemical energy storage : electrical, chemical and thermal effects," Journal of the Franklin Institute, vol. 327, no. 6, pp. 983-992, 1990.

[6] S. Birkett and P. Roe, "The mathematical foundations of bond graphs-I. algebraic theory," Journal of the Franklin Institute, vol. 326, no. 3, pp. 329-350, 1989.

[7] S. Scavarda, M. Amara, and E. Richard, "Determination of the output power in terms of output variables using bond graph," IMACS-IFAC Symposium MCTS 91 Modelling and Control of Technological systems, p. 15, 1991.

[8] R. F. Ngwompo, S. Scavarda, and D. Thomasset, "Inversion of linear time-invariant SISO systems modelled by bond graph," Journal of the Franklin Institute, vol. 333, no. 2, pp. 157-174, Mar. 1996.

[9] P. Gawthrop, "Bicausal bond graphs," SOCIETY FOR COMPUTER SIMULATION, vol. 27, pp. 83-88, 1995.

[10] A. Jardin, W. Marquis-Favre, and D. Thomasset, "Bond graph sizing of mechatronic systems: Coupling of inverse modelling with dynamic optimization," in Proceedings MATHMOD 09 Vienna - Full Papers CD Volume, F. B. I. Troch, Ed., Vienne, Autriche, Feb. 2009, pp. 1929-1938, ISBN 978-3-901608-35-3.

[11] W. L. Oberkampf, S. M. DeLand, B. M. Rutherford, K. V. Diegert, and K. F. Alvin, "Error and uncertainty in modeling and simulation," Reliability Engineering \& System Safety, vol. 75, no. 3, p. 333-357, 2002.

[12] V. H. Nguyen, D. Eberard, W. Marquis-Favre, and L. Krahenbuhl, "Tolerance synthesis using bond graph inversion and fuzzy logic," IEEE International Conference on Mechatronics - ICM2013, Feb. 2013

[13] J. C. Helton, J. D. Johnson, W. L. Oberkampf, and C. J. Sallaberry, "Representation of analysis results involving aleatory and epistemic uncertainty," International Journal of General Systems, vol. 39, no. 6, p. 605-646, 2010.

[14] J. Helton, J. Johnson, and W. Oberkampf, "An exploration of alternative approaches to the representation of uncertainty in model predictions," Reliability Engineering \& System Safety, vol. 85, no. 1-3, pp. 39-71, Jul. 2004.

[15] R. E. Melchers, Structural Reliability Analysis and Prediction, 2nd ed. Wiley, Apr. 1999.

[16] N. Wiener, "The homogeneous chaos," American Journal of Mathematics, vol. 60, no. 4, p. 897, Oct. 1938.

[17] R. Ghanem, "Ingredients for a general purpose stochastic finite elements implementation," Comput. Methods Appl. Mech. Engrg, vol. 168, p. pp., 1999.

[18] R. Ghanem and J. Red-Horse, "Propagation of probabilistic uncertainty in complex physical systems using a stochastic finite element approach," Physica D: Nonlinear Phenomena, vol. 133, no. 1-4, pp. 137-144, Sep. 1999.

[19] O. P. Le Maitre, O. M. Knio, H. N. Najm, and R. G. Ghanem, "A stochastic projection method for fluid flow," Journal of Computational Physics, vol. 173, no. 2, pp. 481-511, Nov. 2001.

[20] C. Dinescu, S. Smirnov, C. Hirsch, and C. Lacor, "Assessment of intrusive and non-intrusive non-deterministic CFD methodologies based on polynomial chaos expansions," International Journal of Engineering Systems Modelling and Simulation, vol. 2, no. 1/2, p. 87, 2010.

[21] J. Li and J. Chen, "The principle of preservation of probability and the generalized density evolution equation," Structural Safety, vol. 30, no. 1, pp. 65-77, Jan. 2008.

[22] M. E. Feki, "Analyse et synthèse de tolérance pour la conception et le dimensionnement des systèmes mécatroniques," $\mathrm{Ph} . \mathrm{D}$. dissertation, Ecole Centrale de Lyon, Jul. 2011.

[23] A. Jardin, "Contribution à une méthodologie de dimensionnement des systèmes mécatroniques : analyse structurelle et couplage à l'optimisation dynamique," Ph.D. dissertation, Jan. 2010. 